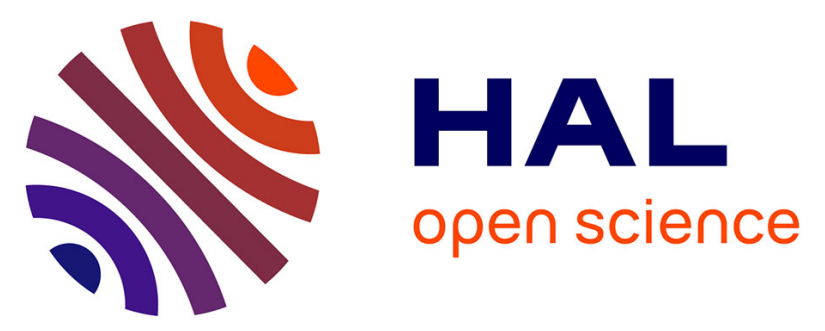

\title{
Tuning graphene transistors through ad hoc electrostatics induced by a nanometer-thick molecular underlayer
}

Ather Mahmood, Cheol-Soo Yang, Seunghun Jang, Lucie Routaboul, Hyunju

Chang, Alessio Ghisolfi, Pierre Braunstein, Laetitia Bernard, Tindara

Verduci, Jean-François Dayen, et al.

\section{To cite this version:}

Ather Mahmood, Cheol-Soo Yang, Seunghun Jang, Lucie Routaboul, Hyunju Chang, et al.. Tuning graphene transistors through ad hoc electrostatics induced by a nanometer-thick molecular underlayer. Nanoscale, 2019, 10.1039/C9NR06407A . hal-02289565

\section{HAL Id: hal-02289565 https://hal.science/hal-02289565}

Submitted on 16 Sep 2019

HAL is a multi-disciplinary open access archive for the deposit and dissemination of scientific research documents, whether they are published or not. The documents may come from teaching and research institutions in France or abroad, or from public or private research centers.
L'archive ouverte pluridisciplinaire HAL, est destinée au dépôt et à la diffusion de documents scientifiques de niveau recherche, publiés ou non, émanant des établissements d'enseignement et de recherche français ou étrangers, des laboratoires publics ou privés. 


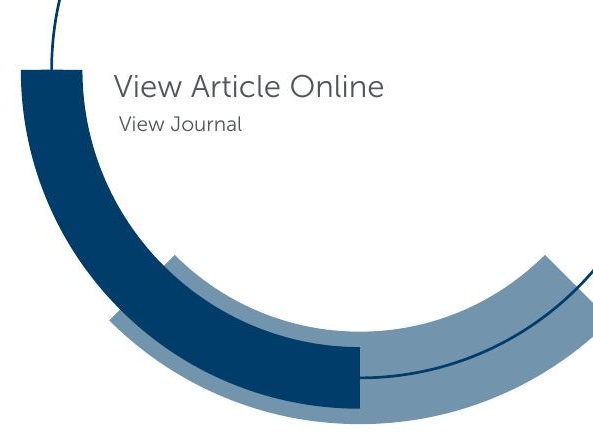

\section{Accepted Manuscript}

This article can be cited before page numbers have been issued, to do this please use: A. Mahmood, C. Yang, S. Jang, L. Routaboul, H. Chang, A. Ghisolfi, P. Braunstein, L. Bernard, T. Verduci, J. Dayen, P. Samori, J. Lee and B. Doudin, Nanoscale, 2019, DOI: 10.1039/C9NR06407A.
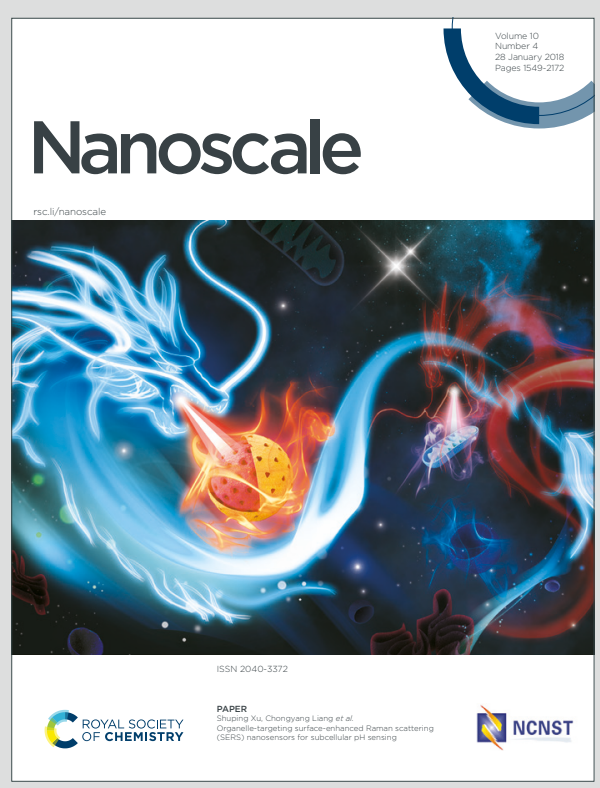

This is an Accepted Manuscript, which has been through the Royal Society of Chemistry peer review process and has been accepted for publication.

Accepted Manuscripts are published online shortly after acceptance, before technical editing, formatting and proof reading. Using this free service, authors can make their results available to the community, in citable form, before we publish the edited article. We will replace this Accepted Manuscript with the edited and formatted Advance Article as soon as it is available.

You can find more information about Accepted Manuscripts in the Information for Authors.

Please note that technical editing may introduce minor changes to the text and/or graphics, which may alter content. The journal's standard Terms \& Conditions and the Ethical guidelines still apply. In no event shall the Royal Society of Chemistry be held responsible for any errors or omissions in this Accepted Manuscript or any consequences arising from the use of any information it contains. 


\title{
Journal Name
}

\section{Tuning graphene transistors through ad hoc electrostatics induced by a nanometer-thick molecular underlayer}

Received 00th January 20xx Accepted 00th January 20xx

DOI: $10.1039 / \times 0 x \times 00000 x$

www.rsc.org/

\author{
Ather Mahmood ${ }^{a} \dagger$, Cheol-Soo Yang ${ }^{b} \dagger$, Seunghun Jang ${ }^{b} \dagger$, Lucie Routaboul ${ }^{c}$, Hyunju Chang ${ }^{b}$, \\ Alessio Ghisolfi ${ }^{d}$, Pierre Braunstein ${ }^{d}$, Laetitia Bernard ${ }^{\text {e, }}$ Tindara Verduci ${ }^{\text {a }}$, Jean-François Dayen ${ }^{\text {a }}$, \\ Paolo Samori ${ }^{f}$, Jeong-O Lee ${ }^{b}$ and Bernard Doudin ${ }^{a}$
}

\begin{abstract}
We report on the modulation of the electrical properties of graphene-based transistors that mirrors the properties of a few nanometers thick layer made of dipolar molecules sandwiched in-between the $2 \mathrm{D}$ material and the $\mathrm{SiO}_{2}$ dielectric substrate. The chemical composition of the films of quinonomonoimines zwitterions molecules adsorbed onto $\mathrm{SiO}_{2}$ has been explored by means of X-ray photoemission and mass spectroscopies. Graphene-based devices are then fabricated by transferring the $2 D$ material onto the molecular film, followed by the top source-drain electrodes deposition. The degree of supramolecular order in disordered films of dipolar molecules was found to be partially improved as a result of the electric field at low temperatures, as revealed by the emergence of hysteresis in the transfer curves of the transistors. The use of molecules from the same family and suitably designed to interact with the dielectric surface yields a disapperance of the hysteresis. DFT calculations confirm that the dressing of the molecules by an external electric field exhibits multiple energy minima landscape that explains the thermally-stabilized capacitive coupling observed. This study demonstrates that the design and exploitation of ad-hoc molecules as interlayer between a dielectic substrate and graphene represents a powerful tool for tuning the electrical properties of the 2D material. Conversely, graphene can be used as an indicator of the stability of molecular layers, by providing insight into the energetics of ordering of dipolar molecules under the effect of electrical gating.
\end{abstract}

\section{Introduction}

In the last decade graphene has emerged as an exciting new material displaying exclusive optical, electrical, thermal and mechanical properties, with potential impact on many realms of science and technology. ${ }^{1}$ Among numerous outstanding characteristics, its highest surface-to-volume ratio makes it extremely sensitive to environmental changes at the nanoscale. The unique electrostatic sensitivity of graphene to nearby molecules is key since it allows to tune the properties of the $2 \mathrm{D}$ material for applications in hybrid molecules-graphene based devices. $^{2}$ It is indeed notorious that the operation and performance of graphene-based solar cells, ${ }^{3}$ organic electronics ${ }^{4}$ and chemical/gas sensors 5,6 critically depend on the interface

a. University of Strasbourg, CNRS, IPCMS UMR 7504, 23 rue du Loess, 67034, Strasbourg, France.

b. Korea Research Institute of Chemical Technology (KRICT), 19 Sinseongno, Yuseong-gu, Daejeon 305-600, Korea.

c. LCC-CNRS, Université de Toulouse, CNRS, UPR 8241, 205 route de Narbonne, 31077 Toulouse Cedex 4.

d. University of Strasbourg, CNRS, CHIMIE UMR 7177, Laboratoire de Chimie de Coordination, 4 rue Blaise Pascal, 67081 Strasbourg, France.

e. Swiss Federal Laboratories fo Materials Science and Technology (EMPA),

Überland Strasse 129, 8600 Dübendorf, Switzerland

f. University of Strasbourg, CNRS, ISIS UMR 7006, 8 allée Gaspard Monge, F-67000, Strasbourg, France.

+ Equal contributors

Electronic Supplementary Information (ESI) available: [details of any supplementary information available should be included here]. See DOI: 10.1039/x0xx00000x between the surface of the electrically conducting 2D material and the (conjugated) organic molecular layers. The vast majority of studies ${ }^{7}$ reported so far have focused on the interactions between graphene flakes supported on a solid substrate and molecules adsorbed on its top in the form of single-molecules, ${ }^{8}$ self-organized thin films $\mathrm{s}^{9,10}$ or supramolecular architectures. ${ }^{11}$ By fabricating planar FET devices, the graphene-substrate electrostatic interactions can possibly impact the transistor properties, with the nearby charges locally doping the graphene layer and shifting the neutrality point. Such electrostatic influence of adsorbed molecules on top of graphene is well-documented. ${ }^{12,13}$

However, what lies underneath graphene also matters. Recent findings revealed that graphene can be 'chemically transparent' since the chemical environment underneath its surface affects the properties of the material grown on its top. ${ }^{14}$ Nevertheless, studies on the inverse FET geometry, based on graphene lying on top of a molecular layer, are much scarcer. To our knowledge, they are limited to functionalized substrates for charge screening purposes, aiming at improving the performance of the transistors by shifting their electrostatically-induced doping (or related work function) $15,16,17$ or to ferroelectric molecular layers designed for driving a very large graphene doping. ${ }^{18}$

We propose to exploit graphene as a tool to explore the properties of an underlying molecular film grown on a dielectric substrate. It 
is also fair to note that the characterization of molecular layers adsorbed onto dielectric surfaces is experimentally much more challenging then when conducting substrates are exploited. This is because surface imaging or spectroscopy tools, including scanning tunneling microcopy, (ultraviolet)photoemission or inverse photoemission spectroscopies, require substrates stables under electric charging.

Here, we investigate ultra-thin films of overall neutral yet dipolar molecules, which can be expected to influence the electronic properties of graphene, bypassing the complications related to the presence of counter-ions associated with charged species. We take advantage of our previous endeavor on the synthesis of a family of zwitterion molecules, their assembly from solution into ultra-thin films, and the study of their physical and chemical properties when adsorbed onto conductive substrates. ${ }^{19,20,21}$ Quinonemonoimines zwitterions (QZW, Scheme 1) are characterized by positive and negative charges on opposite sides of the six-membered ring that are electronically decoupled but chemically connected through carbon-carbon single bonds. $22,23,24$ The overall $12 \pi$ electrons are partitioned in two $6 \pi$-electrons sub-units, with the positive charge delocalized between the nitrogen functions over four bonds involving $6 \pi$-electrons, while the negative charge is likewise spread between the oxygen atoms. This results in a remarkably large electric dipole of typically $10 \mathrm{D}$ across the short planar six-membered ring structure of the benzoquinonemonoimine "core". ${ }^{23}$ We previously detailed how these molecules are prone to undergo spontaneous selfassembly into molecular layer onto gold substrates and show unique electronic properties, making them of interest for enhanced metal-organic interfaces of relevance for organic electronics applications. ${ }^{25}$ Chemical modification of the nitrogen-bound end groups represents a facile strategy for the tuning of their reactivity, packing and electronic properties when adsorbed on metals. ${ }^{19,20,26}$

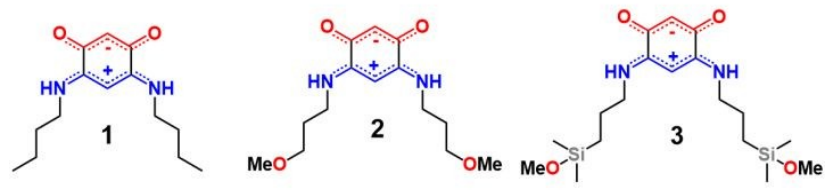

Scheme 1. Chemical structure of molecules 1, 2 and 3.

These dipolar molecules are therefore particularly suited to be interfaced with a graphene surface via electrostatic interactions. The specific choice of the molecular design, which takes full advantage of our developed knowledge, is tailored in order to meet the following characteristics:

- 1 is our best documented member of the family, which has been previously shown to assemble into compact nm-thick films over metallic surfaces. ${ }^{19,20,26}$

- 2 showed better affinity for $\mathrm{Au}$ than $\mathrm{SiO}_{2}$, as revealed by XPS mapping over patterned $\mathrm{Au}$ on $\mathrm{SiO}_{2},{ }^{20}$ and is therefore expected to have small interactions with the
$\mathrm{SiO}_{2}$ substrate.

View Article Online

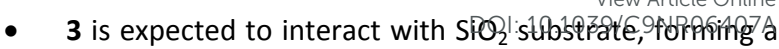
QZW layer featuring a greater stability than $\mathbf{1}$ or $\mathbf{2}$.

The molecular films have been investigated by combining X-ray photoelectron spectroscopy (XPS) and time of flight secondary ion mass spectroscopy (ToF-SIMS) analyses. While the former offers insight into the chemical composition of the adsorbed molecules, the latter provides information on the thickness, quality and integrity of ultra-thin molecular films supported on $\mathrm{SiO}_{2}$ substrates. The electrical characteristics of graphene FET devices built over thin molecular films adsorbed onto such dielectric substrates are exploited as internal gauge to evaluate the stability of such molecular layer, thus using graphene as 'detector' of the underlaying film.

\section{Experimental}

\section{Devices construction}

General procedure for the synthesis of the zwitterions. Commercial 4,6-diaminoresorcinol dihydrochloride and functional amines were used directly without further purification. Solvents were freshly distilled under argon prior to being use. ${ }^{1} \mathrm{H} N M R$ spectra were recorded in $\mathrm{CDCl}_{3}$ on a Bruker $500 \mathrm{MHz}$ instrument, operating at $125 \mathrm{MHz}$ for ${ }^{13} \mathrm{C}$ spectra and $500 \mathrm{MHz}$ for ${ }^{1} \mathrm{H}$ spectra. Chemical shifts are given in $\delta$ units, in parts per million (ppm) relative to the singlet at $\delta=7.26$ for $\mathrm{CHCl}_{3}$. The splittings were designated as s, singlet; $d$, doublet; $t$, triplet; $m$, multiplet; br, broad. Zwitterions $\mathbf{1}$ and $\mathbf{2}$ were readily prepared following published procedures. ${ }^{27,20}$

The synthesis of (6Z)-4-(3-(methoxydimethylsilyl)propan-1amino)-6-(3-(methoxydimethylsilyl)propan-1-iminio)-3-

oxocyclohexa-1,4-dien-1-olate (3) was performed from a suspension of the parent zwitterion $1(0.100 \mathrm{~g}, 0.72 \mathrm{mmol})$ in freshly distilled methanol $(10 \mathrm{~mL})$ to which 3(ethoxydimethylsilyl)propylamine $(0.290 \mathrm{~mL}, 1.52 \mathrm{mmol})$ was added. The solution was heated under argon at $100{ }^{\circ} \mathrm{C}$ for $3 \mathrm{~h}$ (preheated oil bath). After cooling to room temperature, the reaction mixture was filtered and the solid collected was extracted with ethanol. The solution was concentrated under reduced pressure and addition of diethyl ether precipitated the zwitterion as a violet-pink solid which was washed successively with ether and pentane $(0.147 \mathrm{~g}, 51 \%) .{ }^{1} \mathrm{H} \mathrm{NMR}\left(500 \mathrm{MHz}, \mathrm{CDCl}_{3}\right): \delta 0.12(\mathrm{~s}$, $\left.12 \mathrm{H}, \mathrm{Si}-\mathrm{CH}_{3}\right), 0.65\left(\mathrm{~m}, 4 \mathrm{H}, \mathrm{Si}-\mathrm{CH}_{2}\right), 1.77\left(\mathrm{~m}, 4 \mathrm{H}, \mathrm{N}-\mathrm{CH}_{2}-\mathrm{CH}_{2}\right), 3.36$ $\left(\mathrm{m}, 4 \mathrm{H}, \mathrm{N}-\mathrm{CH}_{2}\right), 3.41\left(\mathrm{~s}, 6 \mathrm{H}, \mathrm{Si}-\mathrm{OCH}_{3}\right), 5.14(\mathrm{~s}, 1 \mathrm{H}, \mathrm{N} \ldots \mathrm{C}-\mathrm{C} \mathrm{CH}), 5.43$ (s, $1 \mathrm{H}, \mathrm{O} \cdots \mathrm{C} \cdots \mathrm{CH}$ ), 8.28 (br s, $2 \mathrm{H}, \mathrm{NH}) .{ }^{13} \mathrm{C}\left\{{ }^{1} \mathrm{H}\right\} \mathrm{NMR}(125 \mathrm{MHz}$, $\left.\mathrm{CDCl}_{3}\right): \delta-2.68\left(\mathrm{~s}, \mathrm{Si}-\mathrm{CH}_{3}\right), 13.40\left(\mathrm{~s}, \mathrm{Si}-\mathrm{CH}_{2}\right), 22.42\left(\mathrm{~s}, \mathrm{~N}-\mathrm{CH}_{2}-\mathrm{CH}_{2}\right)$, $45.92\left(\mathrm{~s}, \mathrm{~N}-\mathrm{CH}_{2}\right), 50.47$ (s, Si-OCH$\left.{ }_{3}\right), 80.68$ (s, N……CH), 98.92 (s,

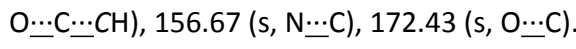

Graphene fabrication. Graphene was grown using low-pressure chemical vapor deposition (LPCVD) methods on a Cu foil (Alfa Aesar, Item No. 46986, 99.8\%, cut into $6 \mathrm{~cm}$ strips) in a hot wall furnace consisting of a 4 in. fused silica tube. Prior to CVD, the foils were cleaned using a $\mathrm{Ni}$ etchant (Transene, TFB) for $5 \mathrm{~min}$ and then 
rinsed with DI water 3 times. A typical growth process flow is as follows: (1) load the $\mathrm{Cu}$ foil, evacuate, heat to $1000{ }^{\circ} \mathrm{C}$, and

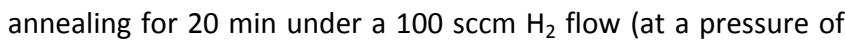
about 70-80 mTorr); (2) introduction of $30 \mathrm{sccm} \mathrm{CH}$ and $30 \mathrm{sccm}$ $\mathrm{H}_{2}$ for 40 min (60 mTorr); (3) cooling down the furnace to room temperature in vacuum, after exposure to $\mathrm{CH}_{4}$ for $40 \mathrm{~min}$. A PMMA solution ( $950 \mathrm{~K}, 4 \%$ by volume dissolved in chlorobenzene) was spin-coated onto the top of the sample at $2000 \mathrm{rpm}$ for $30 \mathrm{~s}$. The PMMA film (thickness of $200 \mathrm{~nm}$ ) was then dried at $30{ }^{\circ} \mathrm{C}$ overnight. The $\mathrm{Cu}$ under the graphene film was etched using a copper etchant solution and washed with deionized water 3 times. The resulting PMMA/graphene layer was collected and transferred on a $\mathrm{SiO}_{2}$ substrate functionalized with QZW molecules. Finally, the PMMA film was dissolved using acetone. Graphene etching is achieved by Ar ion bombardments that are accelerated at $400 \mathrm{~V}$ from the initial gas introduced with a flow rate of $3 \mathrm{sccm}$ at a pressure of $10^{-4}$ mbar. In contrast, Ar ion etching, being more directed and less diffusive, resulted into functional patterned devices.

Molecular films growth. $10 \mathrm{mM}$ solutions of dichloromethane solution of molecules 1-3 were processed at surfaces following two methods. In method 1 , the $\mathrm{SiO}_{2} / \mathrm{Si}$ substrate was rinsed with dichloromethane. The molecular film was prepared by spincoating: a drop the QZW solution was applied onto the basal plane of the surface, then the sample was spun at $2000 \mathrm{rpm}$ for $30 \mathrm{~s}$. This process was repeated 3 times. In method 2, the $\mathrm{SiO}_{2}$ substrate was cleaned with dichloromethane and immersed in the QZW solution for about $18 \mathrm{~h}$ in a sealed container. When the substrate was taken out, dichloromethane quickly evaporated and an uneven layer of zwitterion molecules was observed by optical microscopy. The functionalized substrate was then rinsed with a highly diluted QZW solution (conc $=0.5^{\sim 1} \mathrm{mM}$ ) in dichloromethane to remove the excess zwitterion molecules, and then washed by sample spinning at $500 \mathrm{rpm}$ for $10 \mathrm{~s}$ followed by $3000 \mathrm{rpm} 30 \mathrm{~s}$. This process was repeated twice. XPS analysis of the films of molecules 1, 2 prepared using the two different methods gave similar results. Atomic force microscopy imaging revealed covered substrates of limited roughness. More specifically, while the pristine $\mathrm{SiO}_{2}$ substrates revealed a root-mean square roughness ( $R_{R M S}$ ) of 0.15 $\mathrm{nm}$ as determined on areas of $2 \times 2 \mu \mathrm{m}^{2}$, upon absorption of the molecular layer the $R_{\text {RMS }}$ was found being below $1 \mathrm{~nm}$ for molecules 1,2 and below $2 \mathrm{~nm}$ for molecules 3 .

Graphene transistors fabrication. The chosen graphene transistors architecture comprises a bottom-gate, based on $\mathrm{Si}-\mathrm{n}^{++}$ substrate covered by a $300 \mathrm{~nm}$ thick thermally grown $\mathrm{SiO}_{2}$ layer, and top source/drain Au electrodes, $30 \mathrm{~nm}$ thick, evaporated though a shadow mask yielding channels lengths of several hundreds of $\mu \mathrm{m}$ and channel widths of $40 \mu \mathrm{m}$ (Figure $1 \mathrm{a}, \mathrm{b}$ ). For the purpose of investigating how the buried molecular underlayer impacts graphene transistor properties, it is of paramount importance to avoid heating and solvent processing when transferring the graphene layer. We therefore employed a recently

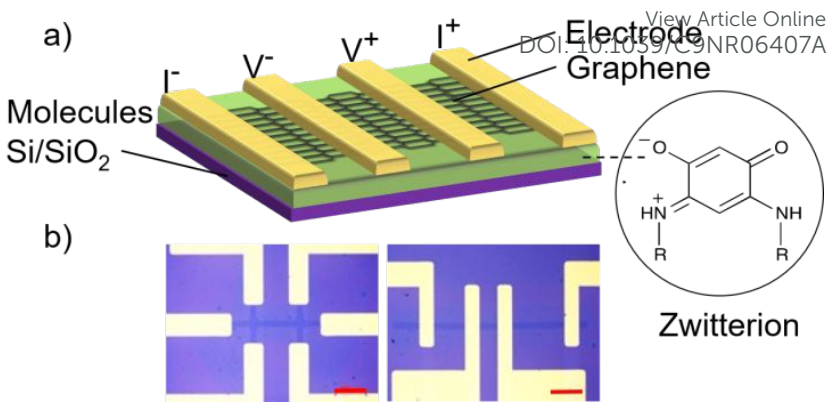

Figure 1. Design of the samples a): Schematics of the graphene-over-molecules device; b): Optical micrograph of typical devices investigated (scale bar is $200 \mu \mathrm{m}$ long).

developed solvent-free approach. ${ }^{28}$ To cross-check the quality of the transferred CVD graphene, we used Raman spectroscopy which revealed that our transistors comprise uniform graphene (mostly bilayer) and exhibit good electrical properties (mobility > $1000 \mathrm{~cm}^{2} \mathrm{~V}^{-1} \mathrm{~s}-1$, clean quantum hall plateau at low temperatures). ${ }^{28}$

Surface spectroscopy characterization. In order to reveal and quantify the presence of thin molecular films adsorbed onto $\mathrm{SiO}_{2}$, two complementary methods were used. XPS Measurements were performed by using the Thermoscientific K-Alpha XPS system (Al Ka $1486.6 \mathrm{eV}$ ). All data were calibrated to a C-C bond energy of $284.9 \mathrm{eV}$. The beam spot size was focused to $400 \mu \mathrm{m}^{2}$ at the takeoff angle of $90^{\circ}$. A pass energy of $20 \mathrm{eV}$ was implemented and energy step size of $0.05 \mathrm{eV}$ was used. The acquisition time was set to $200 \mathrm{~ms}$ and 10 scans of data were recorded, with measurements performed on two regions of the sample. All measurements were conducted at pressures $<6 \times 10^{-8}$ Torr. XPS spectra were recorded on samples prior and after the deposition of the graphene films. ToF-SIMS measurements were performed with an instrument from IONTOF GmbH, Germany. Mass spectra were acquired using $25 \mathrm{keV} \mathrm{Bi}_{3}{ }^{+}$primary ions in high mass resolution mode ( 5000) under the static limit $\left(<10^{12}\right.$ ions $\left./ \mathrm{cm}^{2}\right)$. Positive secondary ions in the mass range 1-800u were recorded on randomly selected areas of $200 \times 200 \mu \mathrm{m}^{2}$. To ensure reproducibility of the results, two measurements were performed on each sample and compared. Cross-check measurements with same operating parameters were carried out on pure bulk molecules (compressed powder) and on bare silicon substrates.

\section{Results and discussion}



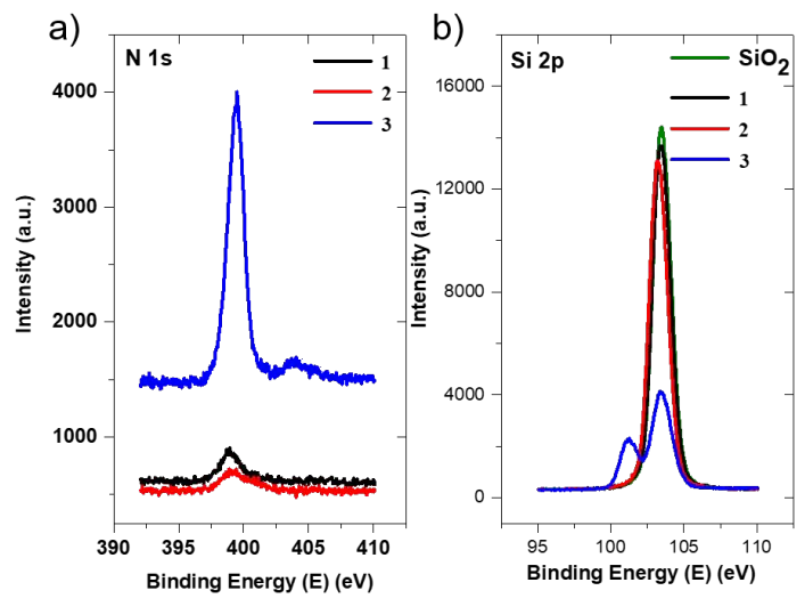

Figure 2. a): XPS N1s spectra of molecules 1, 2, 3 (black, red and blue), respectively over $\mathrm{SiO}_{2}$ substrates. Data shown after the graphene over-layer transfer are identical to those prior to addition of graphene; b): XPS Si $2 p$ spectra of molecules $\mathbf{1}$, 2, 3, respectively over $\mathrm{SiO}_{2}$ with the same color notation as left figure. The data in green color is taken on bare $\mathrm{SiO}_{2}$ substrate.

We first took great care to ensure and quantify the presence of compact molecular thin films on the dielectric substrate. Figure 2 shows XPS spectra measured from all three molecules deposited on $\mathrm{SiO}_{2}$ substrate. In contrast to bare $\mathrm{SiO}_{2}$ and graphene/ $\mathrm{SiO}_{2}$ substrates exempt of detectable XPS signal of $\mathrm{N}$, we observed that deposition of 1-3 on $\mathrm{SiO}_{2}$ resulted in detectable N1s peak, which cannot be attributed to the solvent (Figure 2a). Successive rinsing with ethanol (up to 5 times with $10 \mathrm{~s}$ immersion in solvent) did not noticeably modify the XPS $\mathrm{N}$ peak, providing unambiguous evidence for the stability of the molecular films. Importantly, no noticeable difference in the N1s spectra were found on sample prior or after graphene deposition.

The Si2p background peak was also recorded for 1-3 on $\mathrm{SiO}_{2}$ and compared to the data on as-prepared initial $\mathrm{SiO}_{2}$ substrate (Figure $2 b)$. As expected, the Si2p peak at $103.5 \mathrm{eV}$ decreases when substrates are coated with the QZW films of molecules $\mathbf{1}$ and $\mathbf{2}$, with a further decrease for $\mathbf{3}$, also showing additional spectroscopic features, corresponding to the energy of bulk $\mathrm{Si}$ atoms, likely to result from those of molecule 3 .

From the literature XPS absorption of self-assembled monolayer, revealing an extinction length of $4 \mathrm{~nm},{ }^{29}$ we estimate that molecules 1,2 form layers thinner than $1 \mathrm{~nm}$, while molecules 3 make thicker films, around $4 \mathrm{~nm}$ in thickness.

Further insight into the presence of the molecules of interest on the support was gained by the use of ToF-SIMS that offers both elemental and molecular information of surfaces with ppm sensitivity. Hence, it enables to detect full molecular species of molecules 1, 2 and $\mathbf{3}$ on $\mathrm{SiO}_{2}$, demonstrating their presence and integrity on surfaces. Figure 3 (top) shows part of the positive spectrum recorded on a molecular film of $\mathbf{3}$. Four characteristic peaks can be observed in the mass region of the full molecule. The
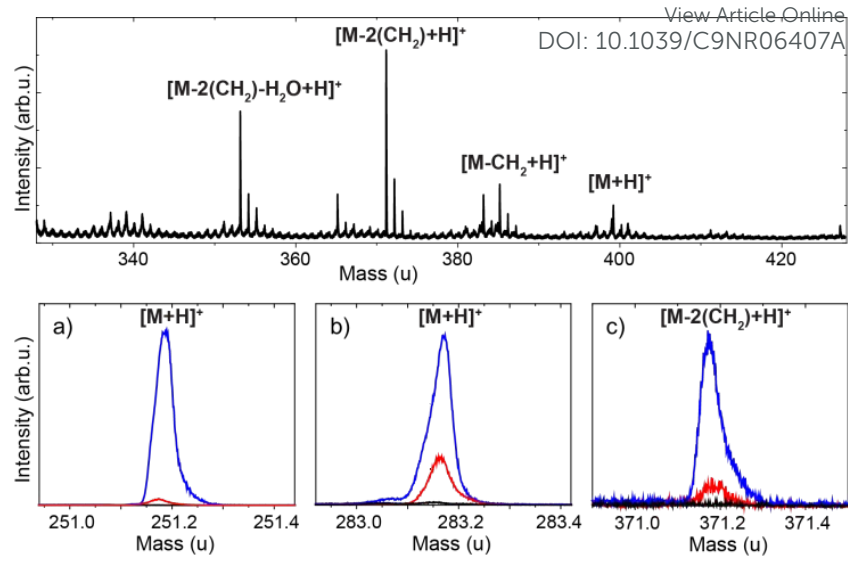

Figure 3. Top: ToF-SIMS positive mass spectrum of molecule 3 adsorbed on $\mathrm{SiO}_{2}$, in the region of molecule $\mathbf{3}$ atomic mass ([M]). Target molecule with and without its methyl terminations and byproduct are observed. Bottom: ToF-SIMS positive mass spectra of molecules $\mathbf{1}$ a), $\mathbf{2}$ b) and $\mathbf{3}$ without the methyl terminations $\mathrm{c}$ ), adsorbed on $\mathrm{SiO}_{2}$ (red), as compared to the references spectra of bare $\mathrm{SiO}_{2}$ (black) and bulk respective molecules (dashed blue).

far-left peak, at mass $399 \mathrm{u}$, corresponds to the full molecule plus one hydrogen $\left([\mathrm{M}+\mathrm{H}]^{+}\right)$. This confirms the presence of the molecule $\mathbf{3}$ on the substrate. Additionally, one can see another peak at mass $385 \mathrm{u}$ and a dominant peak at mass $371 \mathrm{u}$. These correspond to the molecule $\mathbf{3}$ after the loss of one and both methyl termination(s) respectively. Remarkably, this confirms that the molecule 3 spontaneously releases the methyl groups to form Si$\mathrm{O}$-Si bonds with the substrate. Further, the relative intensities of these three peaks demonstrate that the latter binding scheme is the dominant one.

The bottom part of Figure 3 displays zoom-ins on the main molecular ion $\left([\mathrm{M}+\mathrm{H}]^{+}\right)$of the molecules $\mathbf{1}(\mathrm{a}), \mathbf{2}$ (b) and $\mathbf{3}$ (c). The results for SAM samples (red traces), bulk molecules (dashed blue traces) and substrate reference (black traces) are presented and compared. In all three cases, the peak is found to be characteristic (large intensity from the bulk molecule and no intensity from the substrate reference) and demonstrates the presence and integrity of the molecule on the $\mathrm{SiO}_{2}$ substrate. Note that due to different ionization potential of different molecular species and the large dependence of matrix effect, the respective intensities cannot be compared nor estimated from the bulk situation.

Overall, the combination of XPS and ToF-SIMS confirms the presence of uniform films with thicknesses below $5 \mathrm{~nm}$, covering the surface of the $\mathrm{SiO}_{2}$ substrate for molecules 1-3, with good confidence that the molecules remain intact after deposition, and a device construction methodology that ensures that the graphene "lid" on the top did not perturb significantly these assemblies.

Top-contacts bottom-gate graphene transistors supported on $\mathrm{SiO}_{2}$ showed strongly $\mathrm{p}$-doped characteristics, with significant hysteresis in the transfer curves, even though the gate voltage was slowly swept (90 mV/s, Figure 4). Hysteresis and 
a)
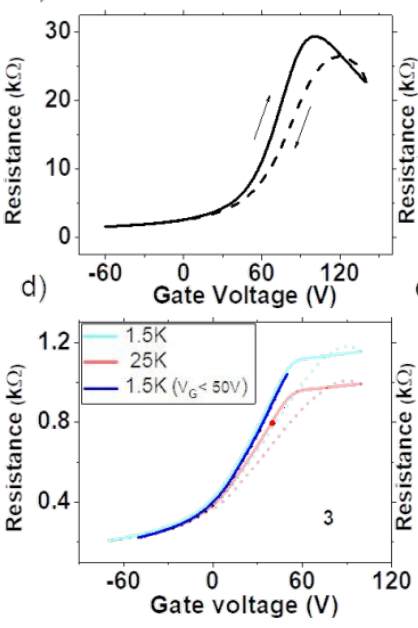

b)

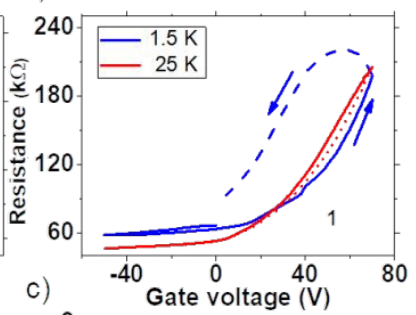

c)

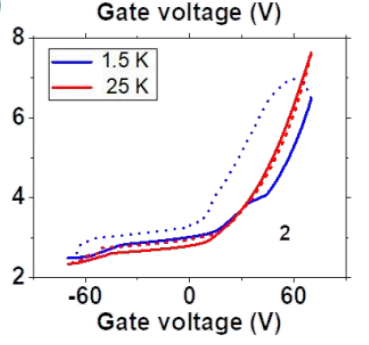

Figure 4. Transistor transfer curves of CVD graphene transferred on $\mathrm{SiO}_{2}$ devices. a): sample transferred over bare $\mathrm{SiO}_{2}$, measured at room temperature, nearly temperature-invariant; b) c) d):devices with substrates covered by QZW molecular layers made of molecules 1, 2, 3 prior to graphene transfer. Hysteresis of the transfer curve is roughly temperature-independent and small for bare $\mathrm{SiO}_{2}$ and requires a larger gating bias to appear. Very large hysteresis occurs at lower temperatures for molecules $\mathbf{1}, \mathbf{2}$, while absent at higher temperatures under moderate gate bias. In contrast, hysteresis similar to bare $\mathrm{SiO}_{2}$ case occurs for molecule 3.

neutral point shift can be reduced by vacuum annealing treatment (down to $10^{-4} \mathrm{mbar}$, with the samples kept at 200 ${ }^{\circ} \mathrm{C}$ ), a procedure previously shown to enhance significantly the quality of the transistors. ${ }^{28}$ Here, we avoided this procedure by preventing heating in order to guarantee the integrity of the adsorbed molecules. We previously showed that CVD graphene devices of high quality can be obtained by proper substrate and post-treatment procedures, ${ }^{28}$ and we traded off here the FET properties for better confidence in the chemical integrity and reproducibility of our devices. The observed deviations from ideal behavior relate to trapped molecules (solvent, oxygen, moisture) and electric-fieldinduced defect in the dielectrics that shift the local density of states of the graphene layer. The so-called positive hysteresis properties of the transfer curves, that shift the neutrality point to more positive gate voltage when sweeping back from large positive bias, persist in vacuum down to $1.5 \mathrm{~K}$ on samples with graphene transferred on bare $\mathrm{SiO}_{2}$ substrates (Figure 4a). This observed hysteresis is commonly attributed to charge trapping in the substrate that imprints local changes in the graphene density of states. ${ }^{30,31}$ For applied gate voltage below $70 \mathrm{~V}$, no hysteresis was observed in the temperature range $300-1.5 \mathrm{~K}$ for the long measurement time scale used.

The capacitive coupling between graphene and nearby charges yields a negative hysteresis behavior: when sweeping back from high positive voltages, the neutrality point is displaced to more negative voltage values. ${ }^{30}$ Such negative hysteresis is usually

explained as resulting from (partial) freezing of the alignment of dipolar molecules in the immediate neighborhodod9 of 9 the 0 ateive graphene layer under the electric field created by the gate voltage. This is for example observed for ion-gated graphene devices. ${ }^{32}$ Dipolar molecular adsorbates, in particular oxygen and moisture,

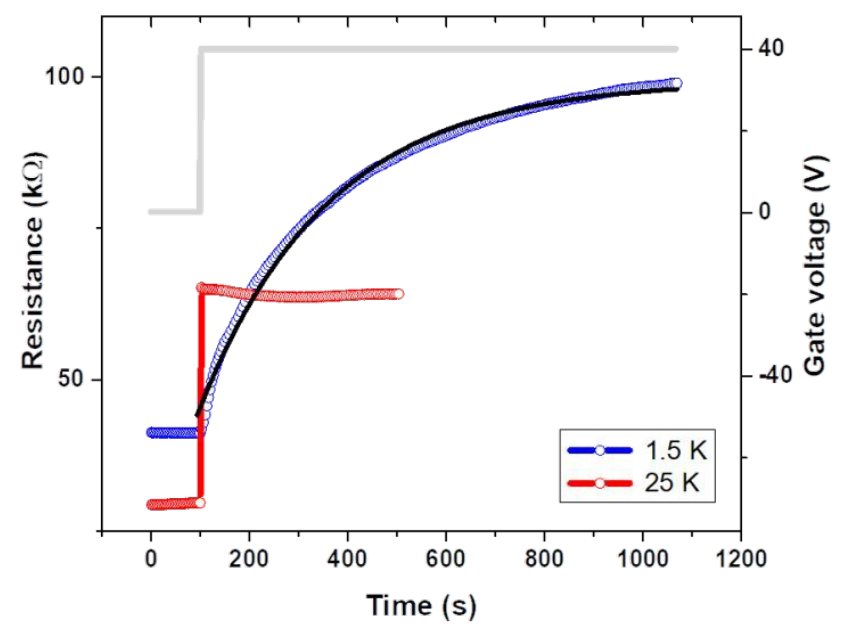

Figure 5. Time evolution of the graphene layer resistance over molecules 1, after a 0 - $40 \mathrm{~V}$ voltage step. The resistance at 1.5 $\mathrm{K}$ varies exponentially, with a fitted time constant of $264 \mathrm{~s}$.

can also induce negative hysteresis. This is well-known with interstitial water, ${ }^{33}$ the hysteresis strongly diminishing below $0{ }^{\circ} \mathrm{C}$, where the electric susceptibility of frozen water becomes negligible.

In our experiments, transistors comprising inter-layers of molecules $\mathbf{1}$ and $\mathbf{2}$ display the appearance of negative hysteresis (Figure $4 \mathrm{~b}, \mathrm{c}$ ). However, this occurs only if the temperature is low enough ( $a$ few $\mathrm{K}$ ), under rather low gate voltage bias (several tens of $\mathrm{V}$ ). Such hysteresis becomes negligible for temperatures greater than $10 \mathrm{~K}$ when a slow gate voltage sweep rate was used. A slow relaxation of the resistance correlates with the observed hysteresis (Figure 5). An exponential slow relaxation of the sample resistance is revealed in the lowest temperature range, with a time constant of typically $10^{2}-10^{4} \mathrm{~s}$ (depending on the temperature and the history of the sample), while absent at higher temperature, where the hysteresis disappears.

One must emphasize that the temperature trend of the negative hysteresis is opposite to the one previously reported in literature. ${ }^{32,33}$ Clearly, the transition temperatures cannot correspond to freezing or a phase transition of (unlikely) solvent residues. Furthermore, negative hysteresis does not occur for the test molecule 3, which was designed to strongly bind to the $\mathrm{SiO}_{2}$ substrate. Indeed, transistors including molecules $\mathbf{3}$ exhibit a small positive hysteresis under larger voltage stress (Figure 4d), which resembles the behavior found on devices without molecules, even though the presence of molecules $\mathbf{3}$ on the substrate is validated by ToF-SIMS and XPS. In essence, low temperatures negative hysteresis unambiguously relates to dipolar QZW molecules weakly bound to the substrate. 
(a)

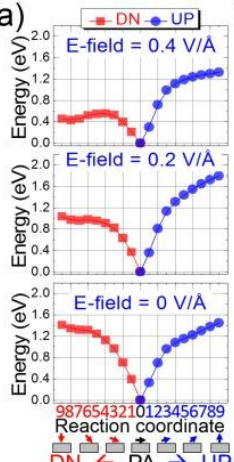

(b)

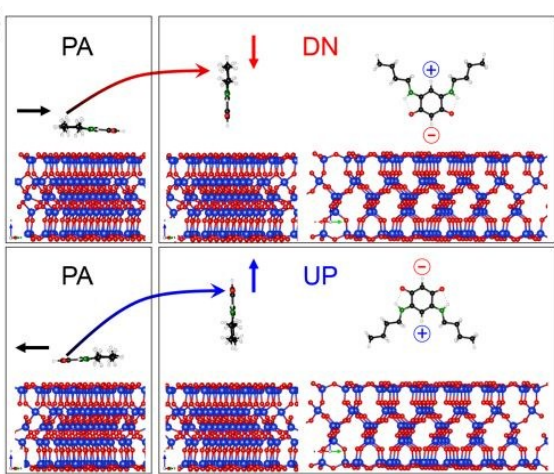

Figure 6. a): Nudged elastic band energy profiles; b): atomic geometries of the rotations of QZW molecule 1 on $\mathrm{SiO}_{2}$, under given applied electric field of $0,0.2$ and $0.4 \mathrm{~V} / \AA \AA$, for the paths from parallel configuration (PA) to 1st perpendicular configuration, where the molecule dipole points down (DN), and from PA to the other perpendicular configuration with dipole pointing up (UP). The red, blue, black, green, and white balls represent $\mathrm{O}, \mathrm{Si}, \mathrm{C}, \mathrm{N}$, and $\mathrm{H}$ atoms, respectively.

Quantitative insight into the energetic of QZW molecules (molecule 1) on $\mathrm{SiO}_{2}$, under applied electric field, was gained by calculations using the Nudged Elastic Band method (NEB). ${ }^{34}$ We simplified our model system to the maximum level, to extract the key points explaining the experimental findings. The atomic geometries of all the model structures were calculated by using the Vienna ab initio simulation package. ${ }^{35,36}$ The exchangecorrelation functional was approximated using the PerdewBurke-Ernzerhof expression. ${ }^{37}$ In particular, the optB86b-vdW functional, implemented in VASP by Klimeš et al. to account for weak van der Waals (vdW) interactions, was used to calculate the optimized structures. ${ }^{38}$ Electron-ion interactions were modelled using the projector-augmented wave method. ${ }^{39}$

The electronic wave functions were expanded in a basis set of plane waves with a kinetic energy cut-off of $400 \mathrm{eV}$.

To compute the initial and final structures needed to perform the NEB calculation, we prepared the reconstructed (001) alphaquartz surface as the $\mathrm{SiO}_{2}$ surface structure. ${ }^{40} \mathrm{Geometry}$ relaxation step in NEB calculation was repeated until the ionic forces were reduced to values below $0.1 \mathrm{eV} / \AA ̊ \AA$. The k-space Brillouin-zone integrations were carried out using only the gamma-point. For all the $\mathrm{SiO}_{2}$ surfaces, the middle $\mathrm{SiO}_{2}$ monolayer was fixed during structure optimization. In all models studied using this calculation, at least 20 Å-thick vacuum regions along the perpendicular direction $(z)$ to the two-dimensional slab were included to minimize interactions between neighbouring image cells.

Figure 6 shows the energy profiles (a) and atomic geometries (b) upon rotating the $\mathrm{QZW}$ molecule on $\mathrm{SiO}_{2}$ from a parallel (face-on) configuration ('PA'), with molecule lying flat on the substrate, to an orientation normal to the substrate with its dipole pointing upwards (configuration 'UP'), or pointing downwards (configuration 'DN'). Non-interacting QZW molecules on $\mathrm{SiO}_{2}$ prefer to lay flat on the substrate, in agreement with previous scanning probe imaging studies performed on Jow coverage

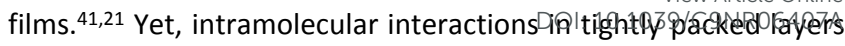
formed by solution processing can exhibit a different behavior, ${ }^{20}$ and it is clear that the adsorption potential energy minimum at low angle values (between molecule and substrate) cannot be applied to our experimental situation. Data of Figure 6 results from energy calculations under an applied electric field of $0,0.2$, and $0.4 \mathrm{~V} / \AA$ respectively, for positive gate bias, estimated to correspond to the largest applied fields in the experiments shown in Figure 5. To maximize the electrostatic interactions, the negative pole of the

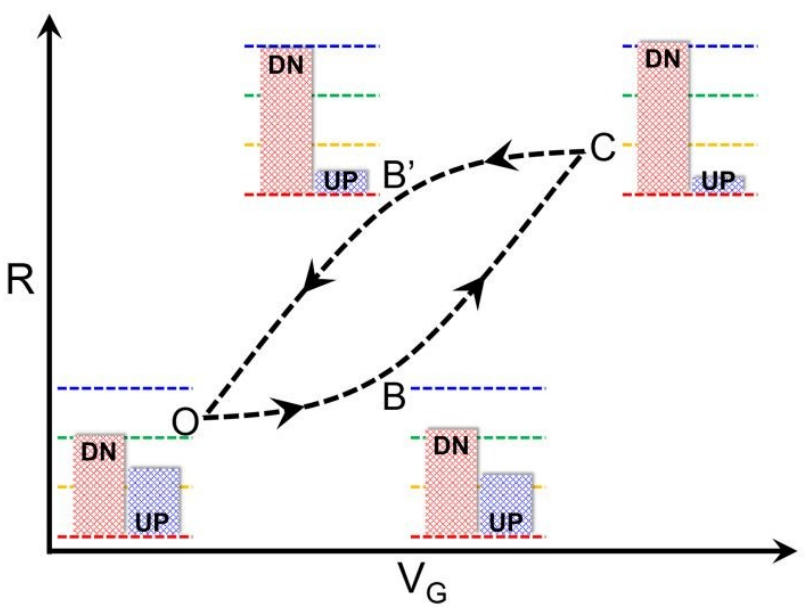

Figure 7. Evolution of dipole population due to the rotation of the QZW molecular on $\mathrm{SiO}_{2}$ under applied electric field. Sweeping the gate voltage relates to the sequence $\mathbf{O} \rightarrow \mathbf{B} \rightarrow \mathbf{C} \rightarrow \mathbf{B}^{\prime}$. In state $\mathbf{O}$, the energy profiles are symmetrical (in Fig. 6) for the two directions of molecules rotations, under the hypothesis of a zero electric field. When increasing the electric field value, the DN orientation is energetically preferred (state B). Upon further increase of the gate voltage makes the DN orientation at nearly $90^{\circ}$ a (meta) stable local minimum of energy. The related reverse energy barrier makes the high angle dressed molecules freeze when sweeping back the gate voltage (state $\mathbf{B}^{\prime}$ ). Estimates of the populations of molecules in DN and UP configurations are included in the figure. This makes the populations of $\mathbf{B}$ different from those of $\mathbf{B}^{\prime}$, the latter having a memory of the populations reached in $\mathbf{C}$.

molecules prefers to point towards to positively biased substrate, explaining the lower energy values when the molecules point downwards.

We propose here that the observed negative hysteresis is due to a difference of the constrained orientation (or 'dressing') of the molecules under electric field. At negligible applied gate voltage (state $\mathbf{O}$ in Fig. 7), the energy configurations for the two rotations $\mathbf{P A} \rightarrow \mathbf{D N}$ and $\mathbf{P A} \rightarrow \mathbf{U P}$ are degenerate. When increasing the gate voltage (state $\mathbf{B}$ in Fig. 7), PA $\rightarrow$ DN is energetically more favorable, and a tendency to dress the molecules downwards emerges. At higher gate bias (state $\mathbf{C}$ in Fig. 7), a stable energy minimum develops for a large angle of the molecules, which can be considered energetically stable in the DN configuration, with an 
energy barrier $\Delta \mathrm{E}$ limiting their probability to escape. This stable statistical occurrence of a population of dipoles pointing down can persist when sweeping back the gate bias voltage, and makes the statistical distribution of the molecules orientations in the state $\mathbf{B}^{\prime}$ differ from the previous state $\mathbf{B}$. The energy barrier $\Delta E$ therefore creates a difference of the conductance state, between $\mathbf{B}$ and $\mathbf{B}^{\prime}$, related to the memory of the dressing in the $\mathrm{DN}$ configuration that occurred at the highest gate voltage applied.

Since the energy barrier $\Delta \mathrm{E}$ is small, being estimated in our calculations as low as $\sim 0.1 \mathrm{eV}$, it is only at the lowest temperature range that the 'reverse' transition path $\mathbf{D N} \rightarrow \mathbf{P A}$ becomes thermally impeded, and the related hysteresis develops. At $25 \mathrm{~K}$, this property disappears, being nullified by the thermally driven motion of the molecules. One can even distinguish a slight overshoot of the resistance value, correlated with the small positive hysteresis observed at $25 \mathrm{~K}$ in Fig 5 . Such thermodynamic 'viscosity' is well known in the field of (nano)magnetism, where a complicated multi-minima energy landscape describes equilibrium magnetic configurations. ${ }^{42}$ Here, we simplified our model to emphasize the two minima energy landscape that explains our findings.

\section{Conclusions}

In summary, our study shows how the electrical read out in graphene-based transistors is a unique tool to gain detailed insight into insulating molecular films deposited over a dielectric substrate. We illustrate this concept on ultrathin films of quinonoid zwitterion molecules 1-3 intercalated between the $\mathrm{SiO}_{2}$ dielectric substrates and the graphene active layer. The transfer curves recorded at the lowest temperatures $(<25 \mathrm{~K})$ reveal that the dipolar alignment of the molecules under gating electric field can be stabilized, for molecules $\mathbf{1 , 2}$ making ultra-thin layers with no indications of strong interactions with the substrate.

Temperature represents also a powerful remote control to modify the electrostatic effect of the molecular film on the device operation: when the temperature exceeds a few tens of $K$ the thermal motions smears out the role played by the presence of the molecules on the transistor device output. However, low temperatures make possible molecular ordering stabilization under electric field, revealed by the graphene 'detector'. Here, the emergence of hysteresis in the transistor transfer curve indicates that such dipolar films made of quinonoid zwitterions can exhibit hysteresis in their polarizability, in analogy to ferroelectrics. Deeper insight into the energetics of the system is needed to clarify if this is a single molecule property or a collective effect, as well as better quantify how graphene plays an active stabilizing role.

Our data details unprecedented temperature dependence properties of graphene transistors; it therefore contributes to better pinpoint the origin of hysteresis in graphene FETs, being a key technological issue requiring better clarifications in the community. It also demonstrates that graphene can be used as an electrical 'detector' of molecular thermal motions,

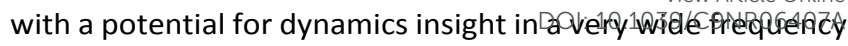
range. Finally, the construction of molecular layers-graphene hybrid systems made it possible to monitor the emergence of novel properties of molecular systems, which are highly relevant for applications in the field of information storage using multi-functional or multiferroic materials.

\section{Conflicts of interest}

There are no conflicts to declare.

\section{Acknowledgements}

This work was supported by the Agence Nationale de la Recherche (FUNGRAPH 11-IS10-003 02, Labex NIE 11-LABX0058_NIE within the Investissement d'Avenir program ANR10-IDEX-0002-02), the International Center for Frontier Research in Chemistry (icFRC), the NRF-ANR program through the National Research Foundation of Korea funded by the Ministry of Education, Science and Technology (NRF_2011K2A1A5-2011-0031552), and the European Commission through the Graphene Flagship Core 2 project (GA-785219). The technical support of the STnano cleanroom is also gratefully acknowledged, especially H. Majjad help for AFM imaging.

\section{Contributions}

J. L., C.Y. and B.D. conceived the experiment, performed by C.Y. and A. M. with S.J. handling the calculations and modeling. L.R., A.G. and P.B synthesized the molecules, H. C. helped for the CVD and J.D. for the transport measurements. L.B., T.V. and P.S. performed the surface spectroscopy studies. All authors contributed to the manuscript writing.

\section{Notes and references}

A. C. Ferrari, F. Bonaccorso, V. Fal'ko, K. S. Novoselov, S. Roche, P. Bøggild, S. Borini, F. H. L. Koppens, V. Palermo, N. Pugno, J. A. Garrido, R. Sordan, A. Bianco, L. Ballerini, M. Prato, E. Lidorikis, J. Kivioja, C. Marinelli, T. Ryhänen, A. Morpurgo, J. N. Coleman, V. Nicolosi, L. Colombo, A. Fert, M. Garcia-Hernandez, A. Bachtold, G. F. Schneider, F. Guinea, C. Dekker, M. Barbone, Z. Sun, C. Galiotis, A. N. Grigorenko, G. Konstantatos, A. Kis, M. Katsnelson, L. Vandersypen, A. Loiseau, V. Morandi, D. Neumaier, E. Treossi, V. Pellegrini, M. Polini, A. Tredicucci, G. M. Williams, B. Hee Hong, J.-H. Ahn, J. Min Kim, H. Zirath, B. J. van Wees, H. van der Zant, L. Occhipinti, A. Di Matteo, I. A. Kinloch, T. Seyller, E. Quesnel, X. Feng, K. Teo, N. Rupesinghe, P. Hakonen, S. R. T. Neil, Q. Tannock, T. Löfwander and J. Kinaret, Nanoscale, 2015, 7, 45984810.

2 K. S. Novoselov, A. K. Geim, S. V Morozov, D. Jiang, Y. Zhang, S. V Dubonos, I. V Grigorieva and A. A. Firsov, 
Science (80-. )., 2004, 306, 666-669. H. Wang, Y. Liu, M. Li, H. Huang, H. M. Xu, R. J. Hong and H. Shen, Optoelectron. Adv. Mater. Rapid Commun., 2010, 4, 1166-1169.

S. Pang, Y. Hernandez, X. Feng and K. Müllen, Adv. Mater., 2011, 23, 2779-2795. W. Yuan and G. Shi, J. Mater. Chem. A, 2013, 1, 10078.

C. Anichini, W. Czepa, D. Pakulski, A. Aliprandi, A. Ciesielski and P. Samorì, Chem. Soc. Rev., 2018, 47, 4860-4908.

M. Gobbi, E. Orgiu and P. Samorì, Adv. Mater., 2018, 30, 1706103.

F. Schedin, A. Geim, S. Morozov, E. Hill, P. Blake, M. Katsnelson and K. Novoselov, Nat. Mater., 2007, 6, 652655.

L. Newton, T. Slater, N. Clark and A. Vijayaraghavan, J. Mater. Chem. C, 2013, 1, 376-393.

N. N. Nguyen, S. B. Jo, S. K. Lee, D. H. Sin, B. Kang, H. H. Kim, H. Lee and K. Cho, Nano Lett., 2015, 15, 2474-2484. M. Gobbi, S. Bonacchi, J. X. Lian, Y. Liu, X.-Y. Wang, M.-A. Stoeckel, M. A. Squillaci, G. D’Avino, A. Narita, K. Müllen, X. Feng, Y. Olivier, D. Beljonne, P. Samorì and E. Orgiu, Nat. Commun., 2017, 8, 14767. X. Wang J.-B. Xu, W. Xie and J. Du, J. Phys. Chem. C, 2011, 115, 7596-7602. A. Matković, M. Kratzer, B. Kaufmann, J. Vujin, R. Gajić and C. Teichert, Sci. Rep., 2017, 7, 9544. S. Chae, S. Jang, W. J. Choi, Y. S. Kim, H. Chang, T. II Lee and J.-O. Lee, Nano Lett., 2017, 17, 1711-1718. Cho, Adv. Mater., 2011, 23, 3460-3464.

K. Yokota, K. Takai and T. Enoki, Nano Lett., 2011, 11, 3669-3675.

N. Cernetic, D. O. Hutchins, H. Ma and A. K.-Y. Jen, Appl. Phys. Lett., 2015, 106, 021603.

Y. Zheng, G.-X. Ni, C.-T. Toh, M.-G. Zeng, S.-T. Chen, K. Yao and B. Özyilmaz, Appl. Phys. Lett., 2009, 94, 163505. J. Xiao, Z. Zhang, D. Wu, L. Routaboul, P. Braunstein, B. Doudin, Y. B. Losovyj, O. Kizilkaya, L. G. Rosa, C. N. Borca, A. Gruverman and P. a Dowben, Phys. Chem. Chem. Phys., 2010, 12, 10329-40.

L. Routaboul, P. Braunstein, J. Xiao, Z. Zhang, P. A Dowben, G. Dalmas, V. Da Costa, O. Félix, G. Decher, L. G. Rosa and B. Doudin, J. Am. Chem. Soc., 2012, 134, 8494-8506.

D. A. Kunkel, J. Hooper, S. Simpson, D. P. Miller, L. Routaboul, P. Braunstein, B. Doudin, S. Beniwal, P. Dowben, R. Skomski, E. Zurek and A. Enders, J. Chem. Phys., 2015, 142, 101921.

A. Sawicka, P. Skurski and J. Simons, Chem. Phys. Lett., 2002, 362, 527-533.

O. Siri and P. Braunstein, Chem. Commun., 2002, 379,

208-9.

Y. Haas and S. Zilberg, J. Am. Chem. Soc., 2004, 126, 8991-8998.

Google Patents, WO2012/025878, 2012.

L. G. Rosa, J. Velev, Z. Zhang, J. Alvira, O. Vega, G. Diaz, L. Routaboul, P. Braunstein, B. Doudin, Y. B. Losovyj and P. A. Dowben, Phys. Status Solidi Basic Res., 2012, 249, 1571-1576.

Q.-Z. Yang, O. Siri, H. Brisset and P. Braunstein, Tetrahedron Lett., 2006, 47, 5727-5731.

A. Mahmood, C. S. Yang, J. F. Dayen, S. Park $M, V_{\text {. }}$, Online Kamalakar, D. Metten, S. Berciaud, الم. O. Leeeand B.06407A Doudin, Carbon N. Y., 2015, 86, 256-263. C. D. Bain and G. M. Whitesides, J. Phys. Chem., 1989, 93, 1670-1673.

30 H. Wang, Y. Wu, C. Cong, J. Shang and T. Yu, ACS Nano, 2010, 4, 7221-7228.

31 G. Kalon, Y. Jun Shin, V. Giang Truong, A. Kalitsov and H. Yang, Appl. Phys. Lett., 2011, 99, 2011-2014. A. Veligura, P. J. Zomer, I. J. Vera-Marun, C. Józsa, P. I. Gordiichuk and B. J. Van Wees, J. Appl. Phys., 2011, 110, 113708. M. Sobrino Fernández, F. M. Peeters and M. Neek-Amal, Phys. Rev. B, 2016, 94, 045436. Henkelman, B. P. Uberuaga and H. Jónsson, J. Chem. Phys., 2000, 113, 9901. G. Kresse and J. Furthmüller, Phys. Rev. B, 1996, 54, 11169-11186. G. Kresse, J. Non. Cryst. Solids, 1995, 192-193, 222-229. J. P. Perdew, K. Burke and M. Ernzerhof, Phys. Rev. Lett., 1996, 77, 3865-3868. J. Klimeš, D. R. Bowler and A. Michaelides, J. Phys. Condens. Matter, 2010, 22, 022201. W. Gao, P. Xiao, G. Henkelman, K. M. Liechti and R. Huang, J. Phys. D. Appl. Phys., 2014, 47, 255301. D. A. Kunkel, S. Simpson, J. Nitz, G. A. Rojas, E. Zurek, L. Routaboul, B. Doudin, P. Braunstein, P. A. Dowben and A. Enders, Chem. Commun., 2012, 48, 7143-7145. E. P. Wohlfarth, J. Phys. F Met. Phys., 1984, 14, L155L159. 


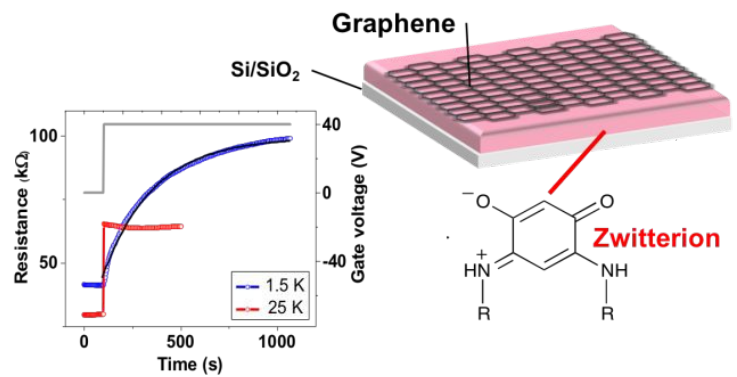

A graphene transistor can reveal ordering of dipolar molecules forming $\mathrm{nm}$-thick underlayer, stabilized under electric field at low temperatures. 